\title{
Modeling of coherent structures in a turbulent jet as global linear instability wavepackets: theory and experiment
}

\author{
Onofrio Semeraro $^{\mathrm{a}}$, Lutz Lesshafft ${ }^{\mathrm{a}}$, Vincent Jaunet ${ }^{\mathrm{b}}$, Peter Jordan $^{\mathrm{b}}$ \\ ${ }^{a}$ LadHyX, CNRS, École polytechnique - Palaiseau, FR \\ ${ }^{b}$ Institut PPRIME, CNRS, Université de Poitiers, ENSMA - Poitiers, FR
}

\begin{abstract}
Perturbation wavepackets in a jet, induced by externally applied forcing, are computed from the linearized equations of motion. An experimentally measured mean flow serves as the base state for this linear analysis, and optimally amplified mono-frequency perturbations are identified, in the sense of maximal gain between the forcing energy input and the flow response energy. Sub-optimal orthogonal forcing/response structures are also discussed. Linear analysis results are then compared to measured perturbation wavepackets in the jet experiment. The study addresses the question to what extent the true dynamics of a turbulent jet can be represented by a model based on linear instability.
\end{abstract}

Keywords:

\section{Introduction}

Since the experiments by Mollo-Christensen [1] and by Crow \& Champagne [2], it has been recognized that turbulent jets exhibit large-scale vortical structures of relatively high spatial and temporal coherence. These structures are well described as wavepackets of synchronized frequency, with streamwise amplitude and phase modulations. It has furthermore been established that the dominant noise radiated from such jets is correlated with these wavepacket structures, as for instance in the numerical study by Freund [3]. Many investigations have since been based on the idea that the

Email address: semeraro [at] ladhyx.polytechnique.fr (Onofrio Semeraro)

Preprint submitted to International Journal of Heat and Fluid Flow

October 12, 2016

(C) 2016. This manuscript version is made available under the Elsevier user license

http://www.elsevier.com/open-access/userlicense/1.0/ 
coherent wavepackets in turbulent jets and other shear flows may be modeled as instability waves (e.g. [4, 5]), linear or nonlinear, that develop in some steady state, either a laminar steady solution of the Navier-Stokes equations or an empirically determined mean flow. Such a model is tempting, because it opens a way for the analysis of the perturbation dynamics and, if the model carries that far, of the sound-producing mechanisms that are responsible for the jet noise.

However, the description of wavepackets in turbulent jets as instability waves within a steady flow state requires an empirical justification. A recent review [6] cites several studies that corroborate the pertinence of such an approach. In particular, Suzuki \& Colonius [7] present sophisticated experimental measurements of near-field pressure fluctuations outside the shear layer of subsonic high Reynolds number jets, and they demonstrate that the coherent fluctuations compare well with the local $k^{+}$instability mode characteristics. Cavalieri et al. [8] take this approach further, by comparing PIV data obtained in the interior of a $M a=0.4$ jet with linear PSE instability calculations. Their study shows remarkable agreement in the development of vortical wavepackets throughout the potential core of the mean flow, for Strouhal numbers between 0.3 and 0.9. At lower Strouhal numbers, and at streamwise distances beyond the potential core, measurements and PSE predictions differ significantly.

The aim of this study is to extend the comparison between experiment and theoretical modeling to a framework of fully global linear stability analysis, named the linear frequency response. Optimization is performed in order to identify the most energy-efficient linear forcing of a jet at a given Strouhal number. New high-quality experimental data, obtained in a jet at $M a=0.9$ and $R e=10^{6}$, is used for a detailed comparison between linear flow-response wavepackets and coherent turbulent structures. A similar investigation by Jeun, Nichols \& Jovanović [9] established good agreement between linear frequency response and LES results for a jet at nominally identical operating conditions. Linear frequency response analysis has been applied in earlier work [10, 11] to different jet configurations. In the present paper, the formalism is applied for the first time to experimental jet data.

The paper is organized as follows. The experimental setup is described in section 2; mean flow quantities are shown, and the main frequency-resolved PIV results are summarized. The frequency response analysis, and some details on numerical procedures, are presented in section 3. Linear analysis results are documented in section 4 and compared to experimental data in 



Figure 1: The "Bruit et Vent" experimental set-up

section 5 .

\section{Experimental setup}

Experiments were conducted at the "Bruit et Vent" jet-noise facility at the CEAT, PPRIME Institute, Poitiers, France. The measurements were carried out at a Mach number $\left(M a=U_{j} / c\right.$, where $U_{j}$ is the jet velocity and $c$ the ambient speed of sound) equal to 0.9 in isothermal conditions. The nozzle diameter $D$ was $0.05 \mathrm{~m}$, giving a Reynolds number of $R e_{D}=\rho U_{j} D / \mu \approx$ $10^{6}$. Transition in the incoming internal boundary layer is triggered using a Carborundum strip glued on the wall upstream from the nozzle exit.

The PIV system consisted of a Photron SAZ camera and a $532 \mathrm{~nm}$ Continuum MESA PIV laser providing $6 \mathrm{~mJ}$ of light pulse energy. The system was placed on a traverse parallel to the jet axis in order to scan the jet flow field from axis location close to the nozzle up to 20 jet diameter. The camera was equipped with a $100 \mathrm{~mm}$ macro lens with low optical distortion, the aperture set at $\mathrm{f} \# 4$. A photograph of the set-up in given in figure 1 .

Two different field of views (FOV) were used during the experimental campaign. The first FOV measured the velocity field in an area of about $2 D \times 2 D$, and was used for axial positions from the nozzle exit up to $x=6 D$. The second FOV measured the velocity field in an area of $4 D \times 4 D$, and was used for more downstream locations, i.e. $x>5 D$. Hence, a finer spatial resolution was obtained for measurements close to the nozzle exit to ensure a good capture of the local velocity gradients. The complete measurement of 
the jet flow was obtained with the use of 11 acquisitions performed at various downstream locations. In between each of these locations an overlap of $20 \%$ of the FOV was set in order to control the correct alignment of the measured velocity fields. A calibration was made at all acquisition positions in order to be able to correct for both the remaining optical distortions and laser light sheet/measurement plane misalignment using a self-calibration procedure [12].

Both the jet flow and the surrounding air were seeded using glycerin smoke particles, whose diameter lays in the range between 1 and $2 \mu \mathrm{m}$, thus sufficiently small to follow the velocity fluctuations of interest in this paper. The particles formed images of 2-3 particles in diameter, and no evidence of peak-locking was found in the data set.

The image acquisition was performed at $20 \mathrm{kHz}(10000$ PIV samples a second) at a resolution of $1024 \times 1024$ pixels. The time between the two laser pulses was set according to the local velocity amplitude and to the laser sheet width (which was set at $2 \mathrm{~mm}$ ), and ranged between 4 and $5 \mu \mathrm{s}$. For each acquisition 42000 image pairs were acquired.

PIV calculations were carried out using a commercial software, and a multi-pass iterative PIV algorithm with deforming interrogation area [13] to account for the local mean velocity gradients. The PIV interrogation area size was set to $32 \times 32$ pixels for the first pass, decreased at $16 \times 16$ pixels for the remaining passes, with an overlap of $50 \%$ between two neighboring interrogation areas. Displacement computed were retained only the correlation peak-ratio was higher than 1.3. After each pass a Universal Outlier Detection (UOD) [14] is applied on a $3 \times 3$ vector grid to avoid corrupted data and enhance the particle motion calculation. Finally, prior to the computation of the flow statistical quantities a 5-sigma filter is applied to remove the remaining outliers and they are replaced using the UOD technique.

\subsection{Mean flow measurements and postprocessing}

The mean flow for which all further analysis will be performed is computed from the PIV measurements through time-averaging. The axial velocity component $\bar{u}$ is presented in Fig. $2 a$, and the correlation $\overline{u^{\prime} v^{\prime}}$ of velocity fluctuations around the mean flow, from which the turbulent viscosity will be estimated, is shown in Fig. $2 b$. The potential core extends about six diameters downstream of the nozzle exit.

Smooth mean flow profiles must be constructed from this data, throughout a large numerical domain, for density, radial and axial velocity. We closely follow the procedure outlined in [15]; in particular, the mean axial 
(a)

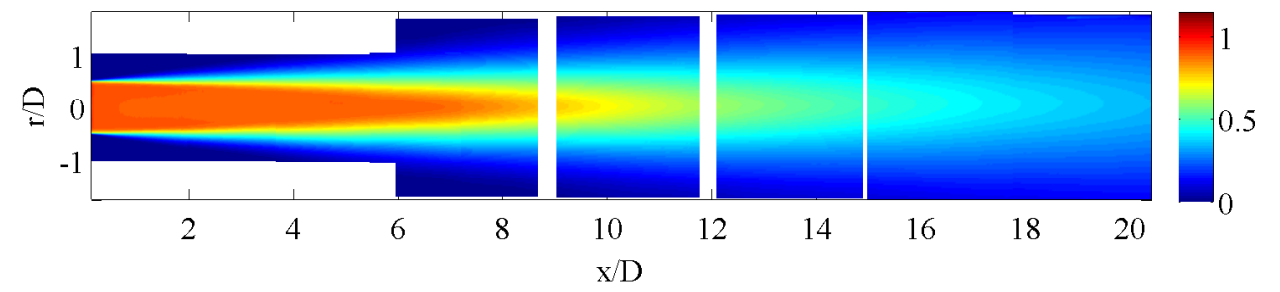

(b)

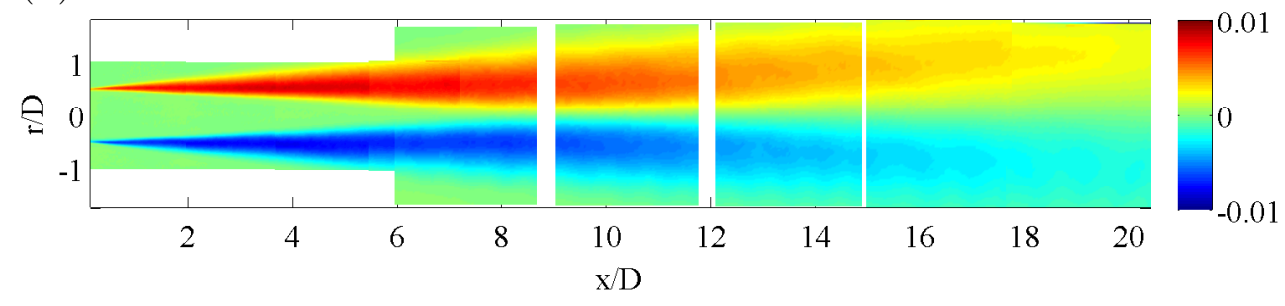

Figure 2: PIV results: the spatial distribution of the meanflow axial velocity and the $\overline{u^{\prime} v^{\prime}}$ fluctuations are shown in $(a)$ and $(b)$, respectively.

velocity is extrapolated in the outer regions $r / D>1$ and $x / D>20$ by use of a Gaussian profile [16], given by

$$
\frac{\bar{u}}{U_{j}}= \begin{cases}1, & \text { if } r<R(x) \\ U_{c}(x) \exp \left(-\frac{(r-R(x))^{2}}{\delta(x)^{2}}\right), & \text { otherwise. }\end{cases}
$$

$R(x)$ characterizes the radial extent of the potential core, $U_{c}(x)$ represents the evolution of the centerline velocity, and $\delta(x)$ marks the radial position along $x$ where $\bar{u}(x, 0)=U_{c}(x) / 2$. Outside the streamwise interval where experimental data is available, the profile parameters $R(x), U_{c}(x)$ and $\delta(x)$ at $x / D>20$ are extracted from a large eddy simulation (LES) of the same setting [17, 18], and the velocity profiles for the pipe flow and the nozzle region are directly taken from this simulation as well. The LES was performed with the solver "Charles" from Cascade Technologies, and it has been shown to reproduce the reference experiment with remarkable precision.

The axial velocity component $\bar{u}$ of the final mean flow model is shown in Fig. 3a. Some smoothing with high-order moving-average filters [19] has been applied for reasons of numerical resolution. The agreement between the experimental data and the reconstructed mean flow model is very satisfactory, 
(a)

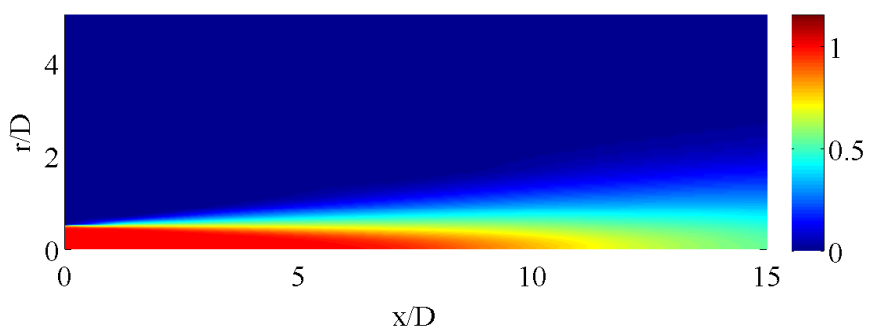

(b)

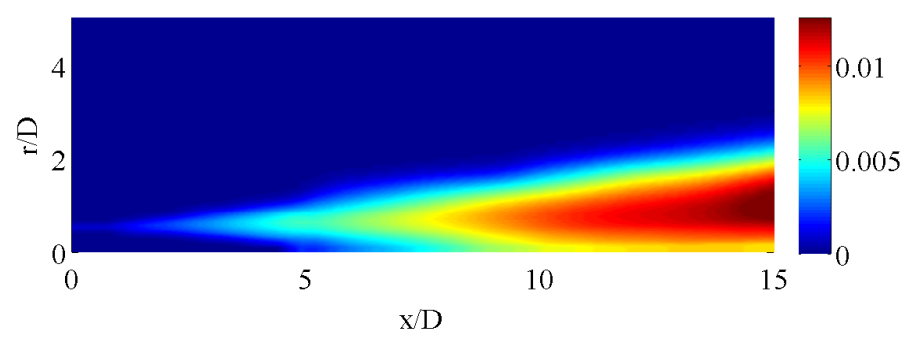

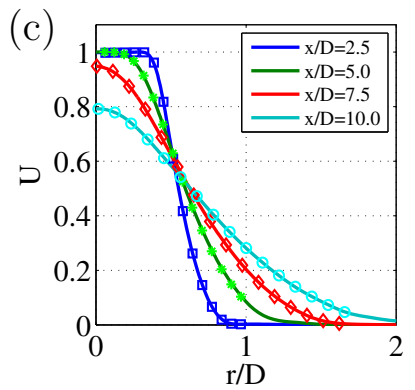

Figure 3: (a) Extrapolated axial mean flow velocity $\bar{u}$ and (b) turbulent viscosity $\nu_{t}$, obtained from processed experimental measurements. (c) Velocity profiles at four different $x$-locations; solid lines: processed mean flow as in $(a)$, markers: experimental data as in Fig. 2a.

as shown in Fig. 3k. The maximum difference between the numerical mean flow from LES and the reconstructed experimental one is approximately $7 \%$ of the velocity $\bar{u}$ in the mixing layer at locations $x / D>10$. Corresponding mean density variations are computed from the Crocco-Busemann relation, and the radial mean velocity $\bar{v}$ is finally obtained from the continuity equation.

\section{Frequency response methodology}

As the jet behaves as an amplifier of external flow perturbations, the appropriate theoretical framework for a global analysis is the frequency response formalism [11], which is synonymously referred to as 'resolvent analysis' or 'input-output analysis' in the literature. This section provides the problem formulation and an outline of the numerical procedure. 


\subsection{Governing equations}

The fully compressible Navier-Stokes equations are written in terms of conservative variables $(\rho, \rho u, \rho v, \rho E)$ in axisymmetric cylindrical coordinates $(r, x)$. Axial and radial velocity components are denoted as $u$ and $v$, respectively, $\rho$ is the density and $E$ the total energy. Only axisymmetric dynamics are considered, therefore all quantities are independent of the azimuthal coordinate $\theta$, and the azimuthal velocity is always zero. The flow quantities are made non-dimensional with respect to the diameter $D$ of the nozzle, the ambient density $\rho_{\infty}$, and the jet centerline velocity $U_{j}$, measured at the nozzle exit $x=0$. The resulting set of equations reads

$$
\begin{aligned}
\frac{\partial \rho}{\partial t}+\nabla(\rho \mathbf{u}) & =0 \\
\frac{\partial \rho \mathbf{u}}{\partial t}+\nabla(\rho \mathbf{u} \otimes \mathbf{u}) & =-\nabla p+\nabla \boldsymbol{\tau} \\
\frac{\partial \rho E}{\partial t}+\nabla(\rho \mathbf{u} E) & =-\nabla \mathbf{h}+\nabla(\boldsymbol{\tau} \mathbf{u}) .
\end{aligned}
$$

The total energy $E$ is defined as

$$
E=\frac{T}{\gamma(\gamma-1) M a^{2}}+\frac{1}{2} \mathbf{u}^{T} \mathbf{u}
$$

with $\gamma=1.4$. The molecular stress tensor is written as $\boldsymbol{\tau}$, and the heat flux is denoted as $\mathbf{h}$. Further details and definitions are given in [20].

Equations (2,4) are linearized around the turbulent mean state presented in Sec. 2.1, in terms of fluctuation variables defined by a Reynolds decomposition, $\rho=\bar{\rho}+\rho^{\prime}$, and accordingly for all other flow variables.

Turbulent viscosity. Our analysis aims at modelling the evolution of coherent fluctuation patterns ('wavepackets') in a fully turbulent jet. Undoubtedly this evolution is affected by the action of fluctuations in the Reynolds stresses, and the question how these effects are to be accounted for in a linear model has been a topic of debate for a long time. A systematic approach has been established [21] on the basis of a triple decomposition, which clearly identified the underlying closure problem. Various strategies have been employed in linear stability studies (useful discussions can be found in [22, 23]), and new approaches have been presented in the recent literature [24, 25], but all of these still rely on empirical models and ad hoc choices. 
For the present study, we opt for a comparably simple turbulent viscosity approach, based on the assumption that the unsteady RANS equations provide a suitable model for low-frequency motion in turbulent jets. The classical turbulent viscosity model, which replaces the Reynolds stresses with a diffusive term as $\overline{u^{\prime} v^{\prime}}=-\nu_{t} \partial_{r} \bar{u}$, has proven to yield accurate results for the steady mean flow of round jets [26]. Here we extend the application of this model to the coherent fluctuations. The turbulent viscosity $\nu_{t}$ is taken to be a function of the coordinates $x$ and $r$, and it is directly obtained from the experimental measurements as

$$
\nu_{t}=-\frac{\overline{u^{\prime} v^{\prime}}}{\partial_{r} \bar{u}+\epsilon}
$$

The original $\overline{u^{\prime} v^{\prime}}$ measurements are shown in Fig. $2 b$. This distribution has been smoothened and extrapolated in a similar fashion as the axial mean flow velocity. Since the mean shear $\partial_{r} \bar{u}$ decays to zero outside the jet, the fraction in Eq. 6] is regularized with a value $\epsilon=10^{-4}$. The resulting distribution of $\nu_{t}$, shown in Fig. 3 $c$, is then added to the molecular viscosity in the linear perturbation equations. Note that $\nu_{t}$ itself is not considered to be a perturbed quantity, as this would require additional modelling assumptions.

\subsection{Computation of optimal forcing/response structures}

Following our initial hypothesis, the harmonic component is obtained as the solution of an optimally forced problem [11]. The discretized state vector is denoted as $\mathbf{q} \in \mathbb{C}^{n}$, where $n$ is the total number of degrees of freedom, describing the amplitude and phase of all fluctuation variables in every point of the numerical domain. The domain lies in the $(r, x)$ plane, and all quantities are taken to be invariant in the azimuthal direction.

The linear perturbation equations are expressed in compact form as

$$
\frac{\mathrm{d} \mathbf{q}}{\mathrm{d} t}-\mathbf{L q}=\mathbf{B} \hat{\mathbf{f}} e^{i \omega t}
$$

On the right-hand side, the system is driven by harmonic forcing $\hat{\mathbf{f}}$ at frequency $\omega$. The operator $\mathbf{B}$ represents a weight function, which is used here in order to place constraints on the forcing. By setting $\mathbf{B}$ to zero everywhere outside the inlet pipe, the forcing is restricted to the pipe interior. This choice is motivated by the observation that the jet dynamics are particularly sensitive to details of the flow upstream of the nozzle, and to the development of the pipe boundary layer in particular [17]. 
Introducing the ansatz $\mathbf{q}=\hat{\mathbf{q}} e^{i \omega t}$, Eq. (7) can be rewritten as the following input-output system

$$
\hat{\mathbf{q}}=(i \omega-\mathbf{L})^{-1} \mathbf{B} \hat{\mathbf{f}}=\mathbf{R}(\omega) \mathbf{B} \hat{\mathbf{f}}
$$

where the resolvent operator $\mathbf{R}(\omega)$ plays the role of a transfer function at a given frequency $\omega$. The forcing $\hat{\mathbf{f}}$ is the unknown of our system: it is defined such that the ratio between the energy of the frequency response and the energy of the forcing input is maximized, i.e.

$$
\sigma_{\max }^{2}=\max _{\hat{f}} \frac{\|\hat{\mathbf{q}}\|_{Q}^{2}}{\|\hat{\mathbf{f}}\|_{Q}^{2}}=\max _{\hat{f}}\left(\frac{\hat{\mathbf{f}}^{\dagger} \mathbf{B}^{\dagger} \mathbf{R}^{\dagger} \mathbf{Q R B} \hat{\mathbf{f}}}{\hat{\mathbf{f}}^{\dagger} \mathbf{Q} \hat{\mathbf{f}}}\right),
$$

where the $\dagger$-superscript indicates the Hermitian transpose. The real positivedefinite matrix $\mathbf{Q}$ represents the norm [see 27]

$$
\|\hat{\mathbf{q}}\|_{Q}^{2}=\int_{\Omega}\left(\rho_{0} \hat{\mathbf{u}}^{2}+\frac{p_{0}}{\rho_{0}}|\hat{\rho}|^{2}+\frac{\rho_{0}^{2}}{\gamma^{2}(\gamma-1) M a^{4} p_{0}}|\hat{T}|^{2}\right) r \mathrm{~d} r \mathrm{~d} x .
$$

The optimal forcing $\hat{\mathbf{f}}$ and associated gain $\sigma_{\max }^{2}$ at frequency $\omega$ are recovered as the leading eigenvalue/eigenvector pair of the operator

$$
\mathbf{C}=\mathbf{Q}^{-1} \mathbf{B}^{\dagger} \mathbf{R}^{\dagger}(\omega) \mathbf{Q R}(\omega) \mathbf{B},
$$

according to the Rayleigh quotient in Eq. (9). This maximum-gain forcing/response pair will be named the optimal mode in the following. All subsequent eigenmodes of $\mathbf{C}$ are orthogonal among each other with respect to the scalar product defined by $\mathbf{Q}$, and they can be ordered according to their real eigenvalues (gains). These forcing/response pairs will be named sub-optimal modes.

The global frequency response analysis describes an externally forced system, and as such it is closely related to the PSE and to the local spatial stability approaches that were used in earlier studies [7, 8]. In contrast to these, the present formalism fully accounts for non-parallelism, and it does not restrict the flow response to locally exponential behavior. The model hypothesis is that the most observable structures in the jet response to stochastic forcing, as measured in the experiment, will correspond to the most amplified wavepackets, as identified in the linear analysis. The acoustic radiation that is associated with near-field wavepackets is obtained as part of the flow response to the applied forcing. 


\begin{tabular}{l|cccc} 
& $\Delta x_{\min }$ & $\Delta r_{\min }$ & $T_{o p t}$ & $\sigma_{\max }$ \\
\hline case A & 0.020 & 0.015 & 60 & 386.77 \\
case B & 0.015 & 0.015 & 60 & 391.90 \\
case C0 & 0.015 & 0.011 & 60 & 394.77 \\
case C1 & 0.015 & 0.011 & 50 & 392.14 \\
case C2 & 0.015 & 0.011 & 70 & 395.13
\end{tabular}

Table 1: Convergence test configurations, run for $S t=0.4$.

\subsection{Numerical methods}

Due to the large dimensions of the discrete linear system, the optimization problem is solved by power iteration, which involves alternate time-stepping of the direct and adjoint perturbation equations. High-order explicit finite difference schemes [19] are used to resolve spatial derivatives. Time integration is performed with a fourth-order Runge-Kutta algorithm. Symmetry boundary conditions are imposed at $r=0$, and convective boundary conditions and sponge regions are used on all other boundaries.

Convergence tests. The numerical domain extends over the interval $x=$ $[-18.5 D, 21 D]$ in the streamwise direction, and over $r=[0,22 D]$ in the radial direction. It is discretized with $\left(N_{r}, N_{x}\right)=(330,625)$ points, concentrated near the nozzle tip with minimum spacings $\Delta x=0.015$ and $\Delta r=0.011$. Grid convergence has been verified by varying the resolution and the point distribution; three examples are reported in Tab. 1, labelled as cases A, B and $\mathrm{C} 0$ for the case $S t=0.4$. The level of convergence is estimated based on the maximum amplification rate $\sigma_{\max }$. In all these test runs, in contrast to the results presented in detail in the following section, the forcing support was not restricted to the pipe interior, and the values of $\sigma_{\max }$ are higher as a result.

The amplification rate is also affected by the time horizon $T_{\text {opt }}$ over which the optimization is carried out. For all results presented in the following section, direct and adjoint time integration is always performed with a time step $\Delta t \approx 0.01$ over $T_{\text {opt }}=60$ nondimensional units; this time horizon corresponds roughly to twice the convection time along the centerline between the nozzle lip and the sponge region. The comparison between cases $\mathrm{C} 0, \mathrm{C} 1$ and C2 (Tab. 1) demonstrates very good convergence. The four most amplified forcing/response pairs are computed at each Strouhal number, using a Krylov space of dimension $N_{k}=15$. 


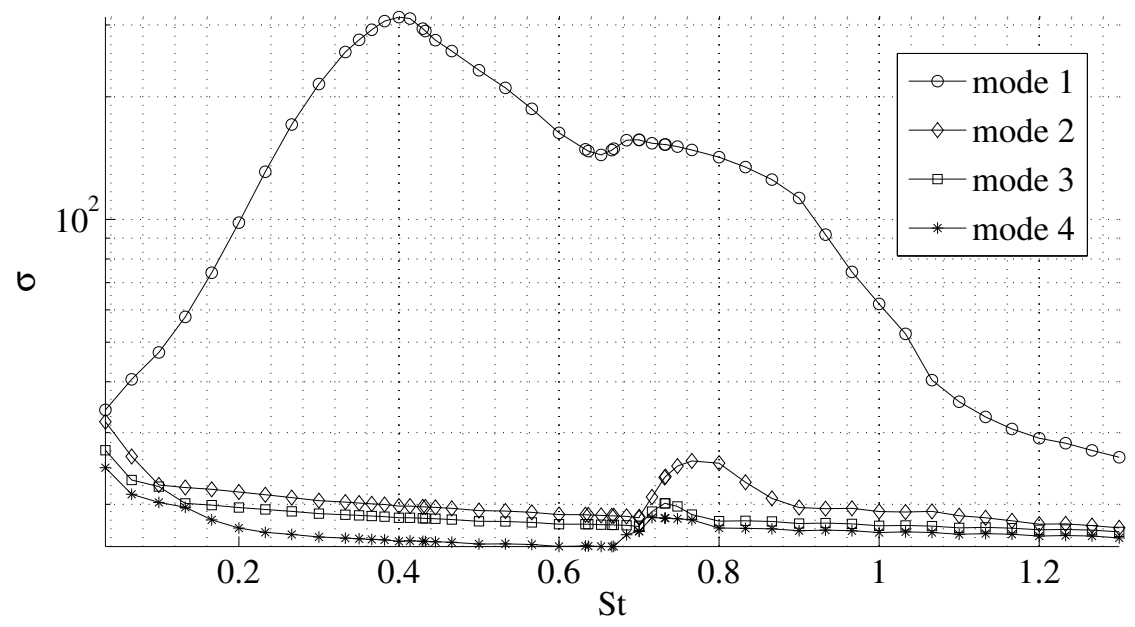

Figure 4: Amplification rate $\sigma$, as a function of the Strouhal number, for the four most amplified modes. The maximum amplification rate is attained at $S t=0.4$. Another local maximum is found around $S t=0.7$ in all four curves.

In order to test the robustness of the results with respect to details of the mean flow, the same computations have also been performed at $S t=0.4$ on the mean flow obtained from LES [17]. Some discrepancies in the flow response arise downstream of the potential core, where indeed the LES and experimental mean flows differ. However, amplification rates and forcing structures are found to be in very good agreement for both mean flows.

\section{Near-field and far-field results of the frequency response analysis}

The amplification gain $\sigma$ is shown as a function of the Strouhal number $S t$ in Fig. 4 for the optimal mode and the first three sub-optimal modes. The overall maximum gain is attained at $S t=0.4$. This value is consistent with previous studies of jets without co-flow: for instance, Crow \& Champagne [2] report a maximum forcing response at $S t=0.3$ in their experiments; Garnaud et al. [11 find the maximum gain around $S t=0.45$ in an incompressible jet, and they show this value to be quite insensitive with respect to the Reynolds number and to the restrictions imposed on the forcing support.

Near its maximum, the gain of the optimal mode is more than one order of magnitude larger than that of all others. The sub-optimal modes exhibit gain values that are comparable among each other over the entire range of Strouhal numbers shown in figure 4. The clear dominance of the optimal over 
the sub-optimal modes is in contrast with the observations of Jeun, Nichols \& Jovanović [9], who found little separation between these in a $M a=0.9$ jet. The mean flow fields used in the present study and in Ref. [9] are believed to be very similar [18]. However, a different norm is used, no Reynolds-stress model is included, and forcing is applied throughout the free-jet region in Ref. 9].

In Fig. 5, the axial velocity component of the optimal forcing, restricted to the pipe interior, is shown alongside the associated response wavepackets for six values of the Strouhal number. The forcing structures are consistently composed of two elements: a plane acoustic wave that travels downstream, and slim vortical structures at the pipe wall that are tilted against the flow direction. The latter structures, which were also identified in incompressible settings [11, resemble optimal perturbations in wall-bounded flows that exploit the Orr mechanism. Such tilted structures rotate as they convect, thereby extracting energy from the mean flow, until they are aligned with the mean flow velocity gradient.

Close to the nozzle, the flow response in the free jet is clearly dominated by a vigorous shear instability. As the mean flow spreads quickly, the perturbations invade the entire jet column. In the fully developed downstream region of the jet, all perturbations eventually decay. Low frequencies sustain spatial growth over a longer streamwise interval, consistent with the local stability properties of a spreading jet [11. Indeed, all optimal response wavepackets resemble each other, except that with growing Strouhal number the characteristic length scales become shorter, affecting both the carrier wavelength and the envelope length.

A peculiar non-monotonic behavior is observed in Fig. 4 in all gain curves around $S t=0.7$. In the optimal mode case (Fig. 5), this coincides with a change in the forcing structure: at Strouhal numbers between 0.7 and 0.8 , the acoustic component of the forcing appears to shift from a plane to an oblique wave pattern, as the half-length of an acoustic wave becomes comparable to the pipe diameter. We hypothesize that these oblique waves in the pipe increase the efficiency of the jet forcing.

Sub-optimal forcing structures and associated flow response wavepackets are presented in Fig. 6. The Strouhal number is 0.4 in all cases, and singular modes $n=2,3,4$ are shown, from top to bottom. The spatial distributions in all three cases are quite distinct from the one of the optimal mode in Fig. 5; in particular, the forcing amplitude peaks in the center of the pipe, although it presents again oblique near-wall structures reminiscent of Orr- 


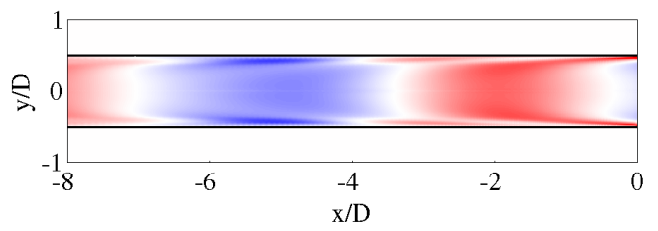

(a) $\mathrm{OF}, \mathrm{St}=0.3$

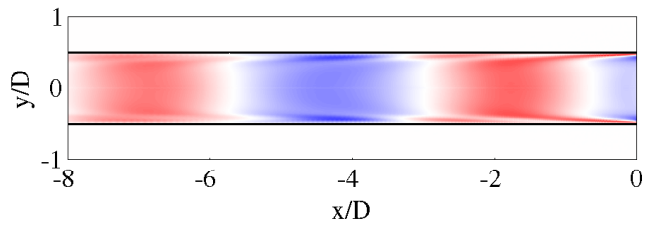

(c) $\mathrm{OF}, \mathrm{St}=0.4$

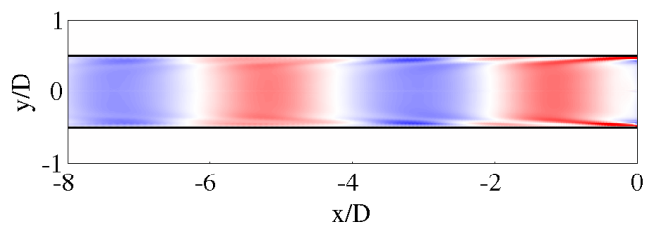

(e) $\mathrm{OF}, \mathrm{St}=0.5$

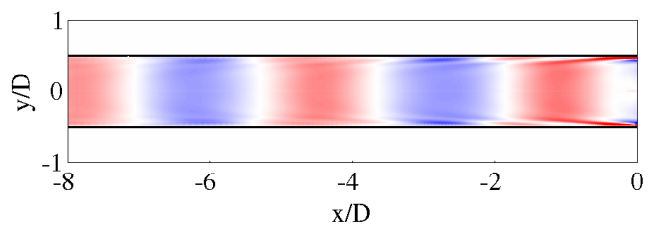

(g) $\mathrm{OF}, \mathrm{St}=0.6$

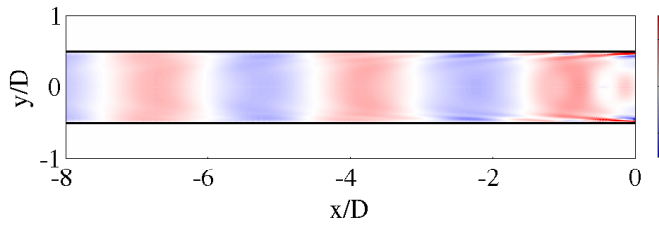

(i) $\mathrm{OF}, \mathrm{St}=0.7$

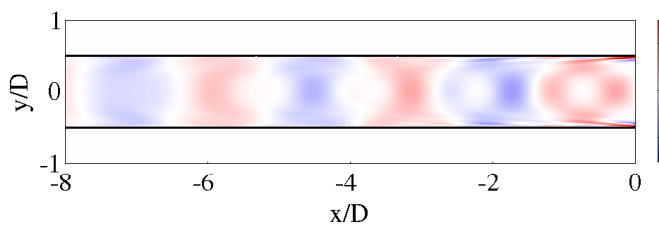

(k) OF, St $=0.8$

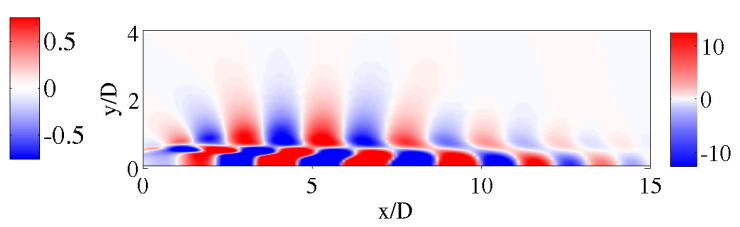

(b) FR, St $=0.3$

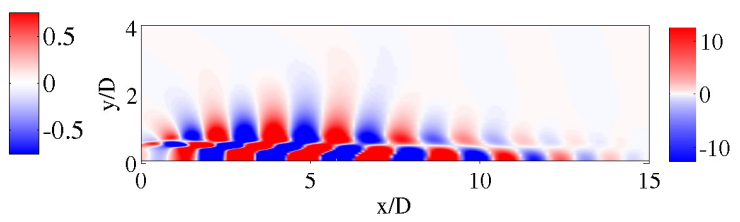

(d) FR, St $=0.4$

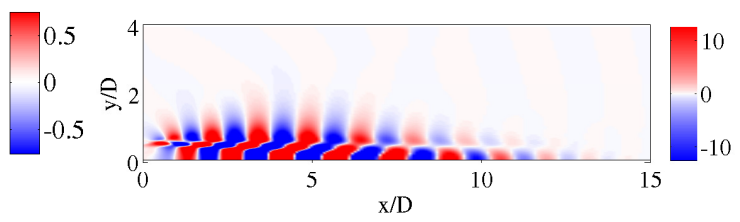

(f) $\mathrm{FR}, \mathrm{St}=0.5$

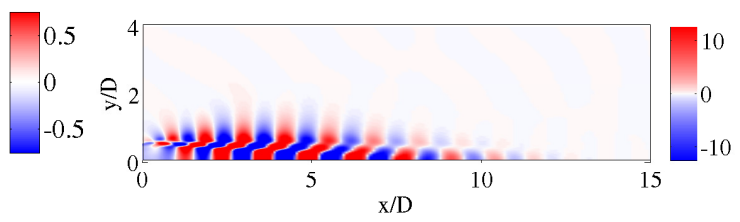

(h) FR, St $=0.6$

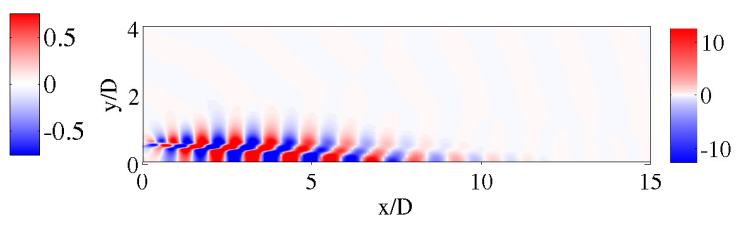

(j) $\mathrm{FR}, \mathrm{St}=0.7$

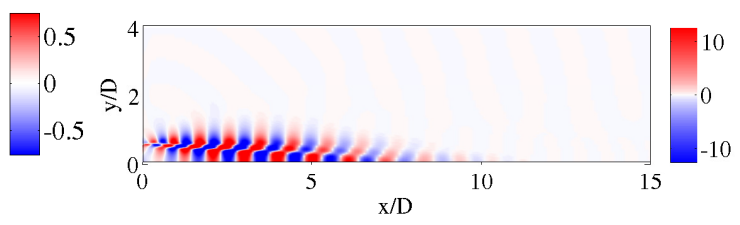

(l) $\mathrm{FR}, \mathrm{St}=0.8$

Figure 5: Optimal forcing $(\mathrm{OF})$ and flow response (FR) structures for various Strouhal numbers. The axial velocity component is shown. The forcing is restricted to the interior of the pipe. 


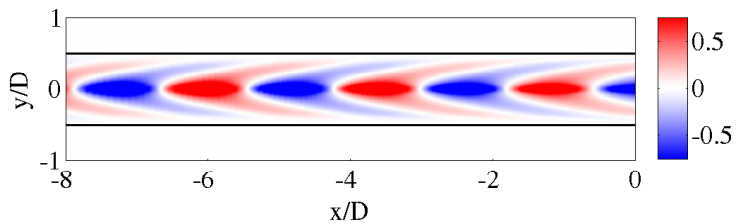

(a) OF, mode 2

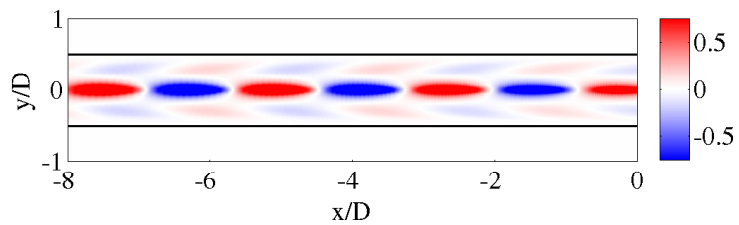

(c) OF, mode 3

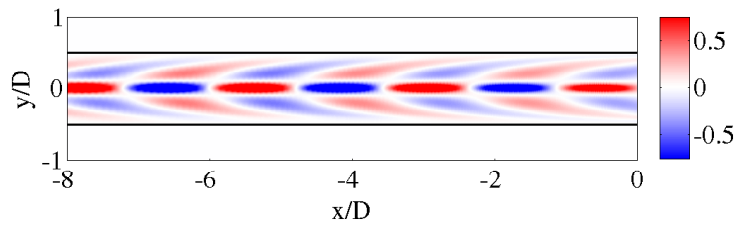

(e) OF, mode 4

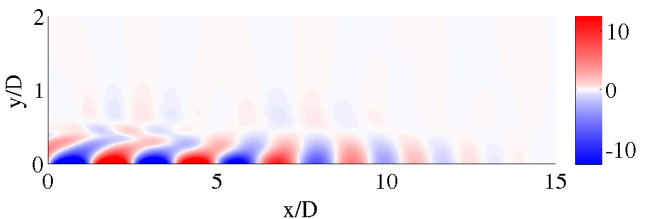

(b) FR, mode 2

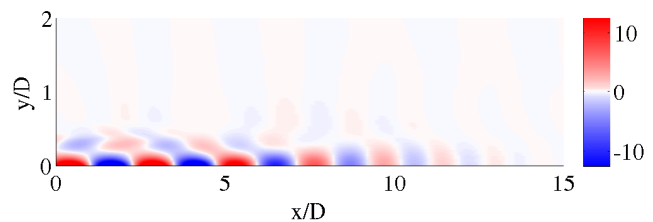

(d) FR, mode 3

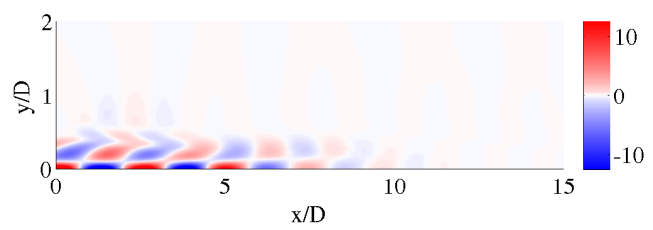

(f) FR, mode 4

Figure 6: Sub-optimal forcing (OF) and flow response (FR) structures for $S t=0.4$ and modes 2,3 and 4 . The axial velocity component is shown. The forcing is restricted to the interior of the pipe.

type forcing, and the wavelength corresponds to a convective (non-acoustic) phase velocity. The forcing structures of the $n=2$ and 4 modes bear a strong resemblance, but mode 4 displays an additional phase change in the radial direction, suggesting that these modes are of the same family, with different radial wavenumbers. The same observation is true for the associated response wavepackets. It is less obvious how the $n=3$ mode may relate to the two others, although it still exhibits similar qualitative features. All three suboptimal modes excite perturbations on the jet centerline, with no discernible shear-type signature. The wavelength of the sub-optimal response modes in the potential core is significantly larger than that of the shear-related optimal mode, and their phase velocities are positive (directed downstream) throughout the near field, including the pipe.

The acoustic far field of the flow response wavepackets is finally visualized in Fig. 7, for the optimal mode at $S t=0.4$ and 0.64. Snapshots of the density perturbation field are shown, with a color scale that resolves the low 

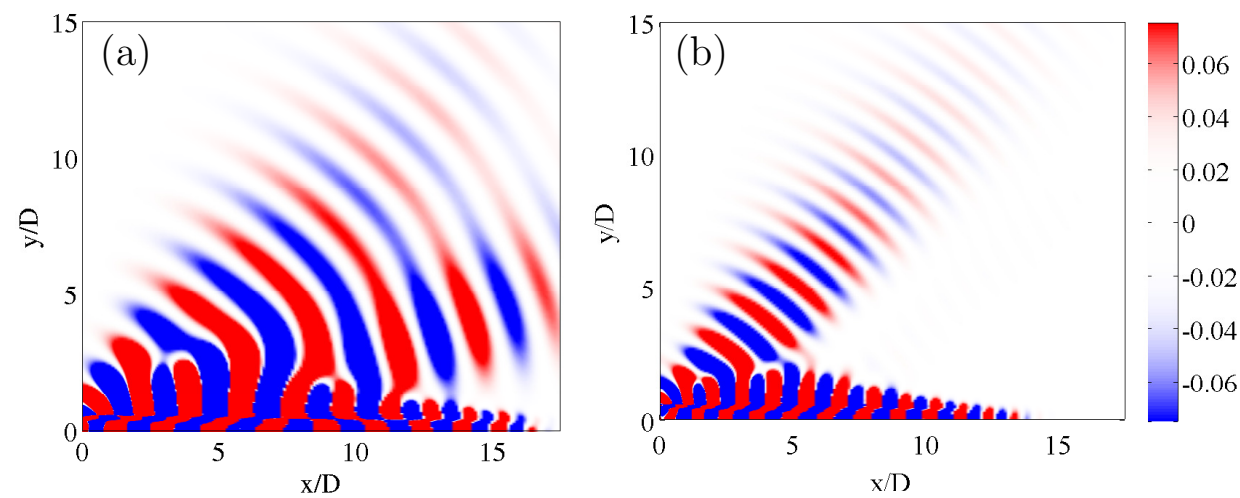

Figure 7: Density fluctuations of the flow response to optimal forcing at $(a) S t=0.4$, and (b) $S t=0.64$.

acoustic amplitudes away from the jet. The sound waves seem to emanate either from the near-nozzle region, where hydrodynamic perturbations grow exponentially, or perhaps directly from the nozzle. A double-beam pattern forms at $S t=0.4$, whereas a single beam is found at $S t=0.64$. Similarly marked directivity patterns are characteristic for the acoustic fields of the optimally forced wavepackets, also at other Strouhal number values.

\section{Comparison with the experiment}

The linear model results can be compared to experimental measurements, provided that the axisymmetric fluctuation component in the experiment can be isolated. A reasonably easy way to do this is to only regard fluctuations of the axial velocity on the jet centerline; in a Fourier decomposition into azimuthal modes, only the axisymmetric mode is non-zero in this velocity component. The centerline velocity fluctuations are readily extracted from the PIV data, and a temporal FFT is performed. The results are presented in Fig. $8 a$ as logarithmic color contours of the power spectral density (PSD) in the $x$-St plane.

Corresponding centerline data, obtained from the frequency response analysis, are shown in Fig. $8 b$ for comparison. The resemblance between the experimental data and the linear model results is not particularly convincing at this point. The linear frequency response appears to underpredict the amplitudes at low Strouhal number, and it does not seem to reproduce a certain anomaly that is found in the experimental spectrum at $S t=0.45$. 
(a)

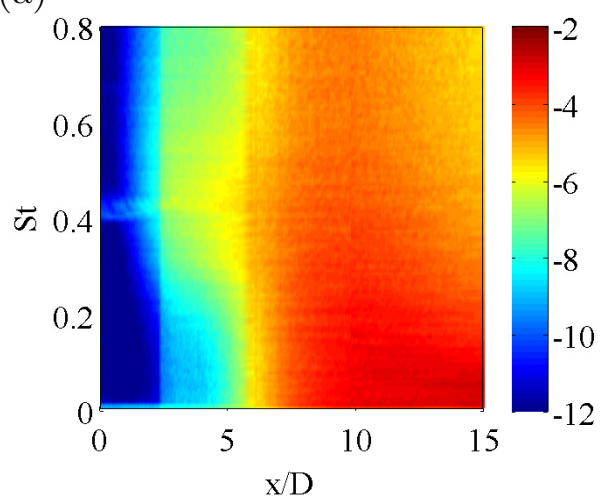

(b)

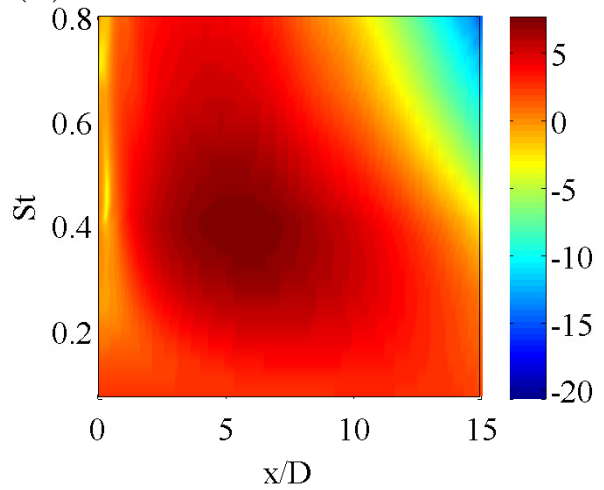

Figure 8: Hydrodynamic near field: power spectral density of axial velocity fluctuations on the jet centerline. (a) PIV measurements; (b) linear frequency response results (axisymmetric mode). A logarithmic scale is used in both figures.

However, all these differences may be due to the amplitude normalization of the frequency response results. In Fig. $8 b$, it is assumed that the forcing energy input is identical at every Strouhal number, which is certainly not the case in the experiment. Furthermore, the time-resolved PIV measurements in the range $2.2 \leq x \leq 3.8$ are affected by optical distortions, which explains an irregularity in the contours in Fig. 8a.

A more pertinent comparison can be made for individual Strouhal numbers. Fig. 9 compares experimental and linear model PSD data for eight values of $S t$ between 0.1 and 0.8 . As the amplitude in the linear model is an arbitrary constant, the frequency response (FR) curves may be vertically shifted for an eyeball fit with the reference data. PIV results in the region $2 \leq x \leq 4.5$ are excluded from these plots. In order to give a more complete picture, LES data [17] are also included.

The linear model fails to capture the experimentally observed wavepackets at low Strouhal numbers, $S t \leq 0.2$. At $S t=0.3$, the initial phase of exponential growth in the LES results is fairly well reproduced, although only over a short streamwise distance. The reference data shows atypical behavior at $S t=0.4$, which again is not reproduced by the linear analysis. At Strouhal numbers between 0.5 and 0.8 , however, the linear predictions compare favorably with experiment and LES over the first three diameters. A sometimes irregular curve shape may seem surprising, but is easily explained by the fact that only centerline values are plotted here, whereas the fluctuations near the nozzle exit are concentrated in the shear layer. 


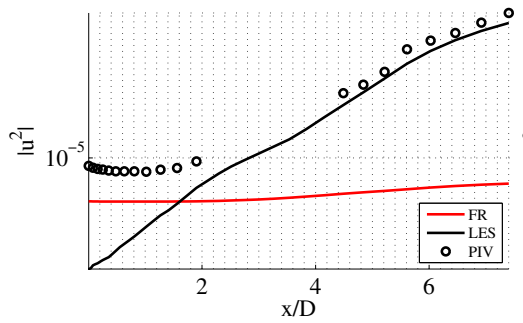

(a) $\mathrm{St}=0.1$

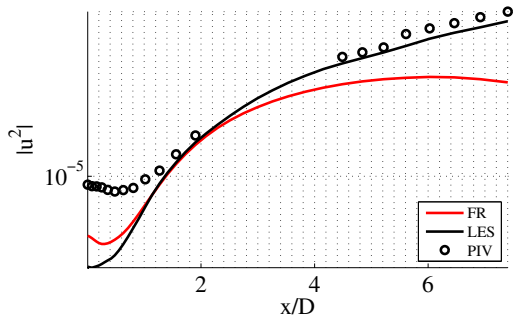

(c) $\quad \mathrm{St}=0.3$

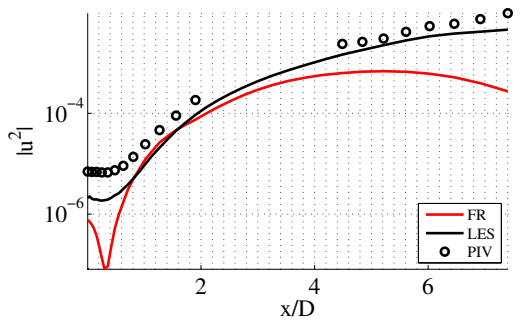

(e) $\mathrm{St}=0.5$

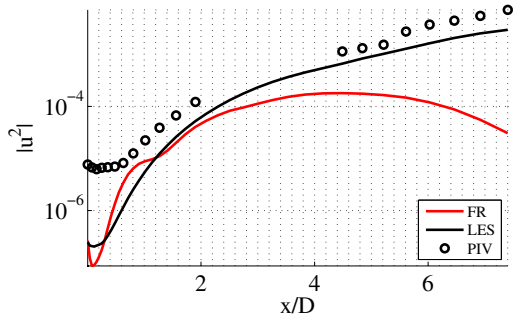

(g) $\quad \mathrm{St}=0.7$

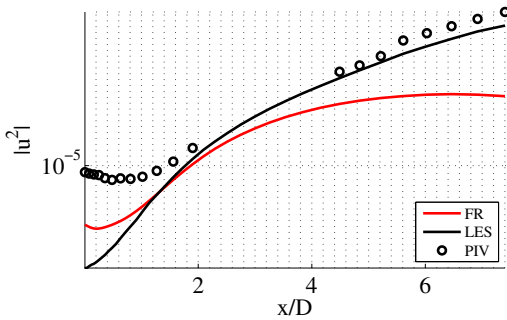

(b) $\quad \mathrm{St}=0.2$

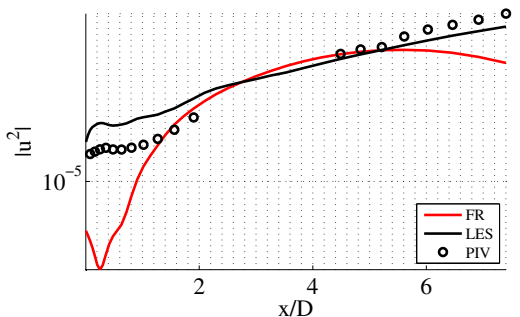

(d) $\quad \mathrm{St}=0.4$

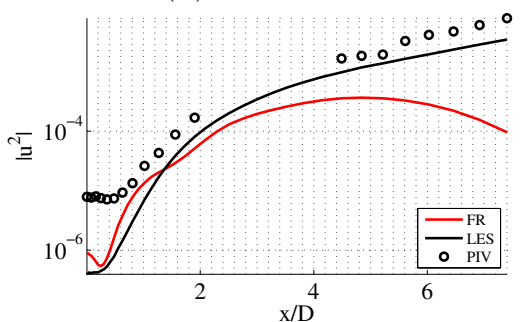

(f) $\quad \mathrm{St}=0.6$

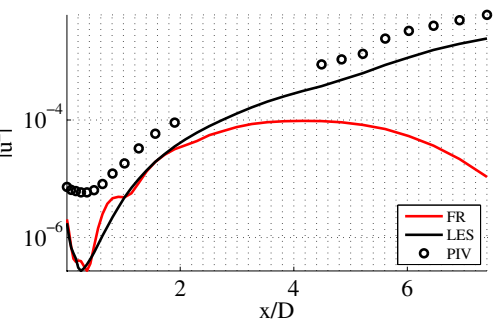

(h) $\quad \mathrm{St}=0.8$

Figure 9: Power spectrum density (PSD) of the axial momentum fluctuation velocity $u^{\prime}$ on the centerline as a function of $x / D$, at various Strouhal numbers, for three different datasets: i) linear frequency response (red, solid line); ii) LES data (black, solid line); iii) PIV data (markers).

Far-field acoustic data is found from the microphone array measurements, taken at 16 azimuthal positions around the jet, at a radial distance of 14.2 
(a)

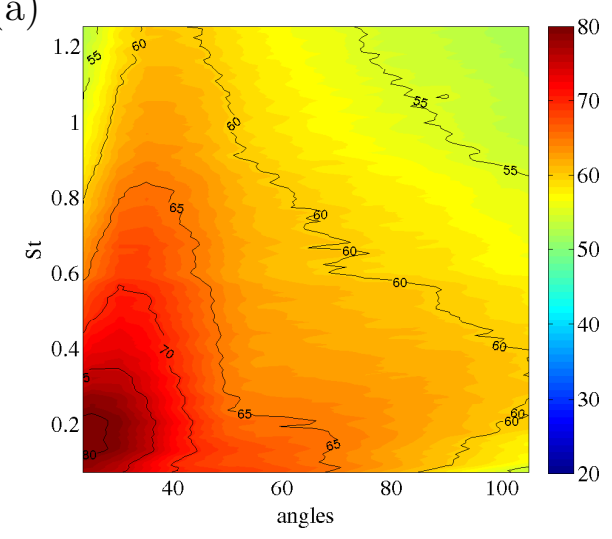

(b)

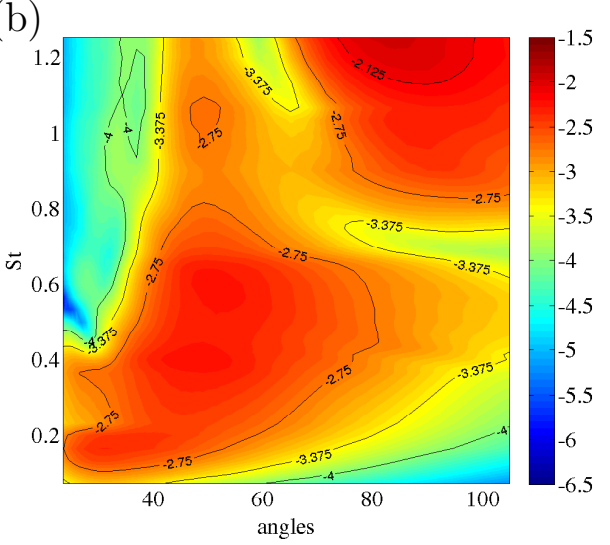

Figure 10: Acoustic far field: power spectral density of acoustic waves outside the jet. (a) microphone pressure measurements (azimuthal average), scaled as $\mathrm{dB} / \mathrm{Hz}$; (b) linear frequency response results (axisymmetric mode), scaled with respect to unit energy forcing input at each Strouhal number.

jet diameters, and at axial positions between $x=-4$ and 39. A cylindrical surface is considered. Averaging over all azimuthal positions yields a good approximation of the instantaneous axisymmetric sound component, which is then Fourier-transformed in time. The results are shown in Fig. 10a as a function of Strouhal number and radiation angle. The angle with the jet axis, measured from the the nozzle exit $x=0$, is simply obtained by rescaling the axial coordinate; the reported values are not scaled with respect to the distance from the nozzle. In experiments at Mach numbers between 0.35 and 0.6 [8], the sound emission associated with the axisymmetric wavepackets is dominant at low angles, below $25^{\circ}$; the present data confirm this trend also at $M a=0.9$. Higher-order azimuthal modes (not considered here) radiate preferably at higher polar angles.

Corresponding data from the linear frequency response analysis are plotted in Fig. $10 \mathrm{p}$ for comparison. It must be noted that the comparison between experiment and linear model again suffers from the arbitrary normalization of the linear results, and a uniform forcing norm of unity has been used in order to generate this figure. As a result, absolute contour values cannot be expected to agree between Figs. $10 a$ and $10 \mathrm{p}$, but the directivity patterns at individual Strouhal numbers can be compared. Both in the experiment and in the linear analysis, one dominant beam is identified at $S t<0.8$, and in both cases its angle increases slowly with Strouhal number. At $S t=0.8$, the maximum radiation is found around an angle of $50^{\circ}$ in the linear model, 
whereas it is closer to $40^{\circ}$ in the experiment. Above $S t=0.8$, a strong additional acoustic lobe appears in the linear results, radiating in the direction perpendicular to the jet, and even upstream. This lobe however is absent in the experiment. Precisely the same behavior was observed by Garnaud et al. [10], who obtained strong upstream radiation in the linear frequency response of a jet at high Strouhal numbers, which was not at all present in the reference DNS.

\section{Conclusions}

A linear frequency response analysis has been performed, for the first time, on a turbulent jet mean flow obtained from experiments. The jet operates at $M a=0.9$ and $R e=10^{6}$. The linear analysis allowed to identify the optimal forcing mode over a range of Strouhal numbers, $0.1 \leq S t \leq 1.2$, as well as the three following sub-optimal forcing modes, together with the associated flow response. Detailed hydrodynamic near-field and some acoustic far-field results have been documented, and they have been compared to experimental data. Near-field results from a companion LES study [17] were included in order to complement the comparison in flow regions where the experimental data was incomplete. The dominant linear mode has been found to agree reasonably well with the nonlinear reference data in the region close to the nozzle, where the linear approximation is valid, at Strouhal numbers between 0.3 and 0.8. The particular value $S t=0.4$ is an exception: here, experimental and LES data both display markedly different trends than at other Strouhal values, and this phenomenon has been described in full detail in recent studies [28] to be the manifestation of 'trapped acoustic modes'. Remarkably, no such trapped modes seem to play a dominant role in the present analysis.

The failure of a linear instability model based on the optimals to predict the flow dynamics at low Strouhal numbers, and several diameters downstream of the nozzle, is consistent with previous studies that used PSE or linearized Euler simulations [5, 29]. However, recent results suggest that it might be possible to overcome some of these limitations within the linear frequency response formalism [25, 30].

The branch of optimal modes exhibits forcing structures that are clearly composed of two distinct components: an acoustic wave (plane for $S t<0.7$ and oblique for $S t>0.7$ ) and oblique vortical waves near the pipe wall that draw their efficiency from the Orr mechanism. Both components trigger 
a shear instability in the potential core region of the jet, which provides the mechanism for strong spatial growth of perturbations. The resulting wavepackets are of the same kind as those obtained, for instance, by Crighton \& Gaster [4], or from PSE [5]. It is noted that the relative strength of acoustic versus vortical forcing components necessarily depends on the norm that is used to measure the input energy. The frequency response formalism involves several choices that affect the results, such as the localization of the forcing, the choice of the norm and the modeling of Reynolds stresses.

A recent frequency response analysis of a $M a=0.4$ jet [30], where turbulent viscosity is not accounted for and no localization restriction is imposed on the forcing, identifies very similar forcing structures of the optimal mode as found in the present results. The sub-optimal modes differ significantly however: without localization, both the sub-optimal forcing and the response structures reside inside the shear layer of the free jet. The present choice to restrict the forcing to the nozzle pipe allowed to identify the influence of the upstream flow system onto the free-jet dynamics [17]. The first three suboptimals in this case carry their maximum amplitude on the centerline of the pipe, and their radial structure suggests that they belong to one family, hierarchically ordered by a radial wavenumber. These centerline perturbations appear to drive jet-column instability modes in the free jet, which inherit the radial structure from the forcing modes. It is interesting to note that all shear-layer instability dynamics seems to be contained in the optimal forcing, whereas it is absent in the subsequent sub-optimals. The frequency response formalism, together with the localized forcing, cleanly separates shear-layer and jet-column dynamics inside the potential core.

A rather strong separation has been noted between the optimal gain value and that of the highest sub-optimal, at least over the relevant interval $0.2 \leq$ $S t \leq 1$. The present results in this respect differ from the findings of Jeun, Nichols \& Jovanović [9]. These differences are attributed to the localization assumption, the turbulent viscosity model and the choice of the optimization norm.

Finally, only a preliminary description of acoustic radiation has been provided in this study. The comparison between linear analysis and experiment is limited to the qualitative directivity of the sound emission associated with the axisymmetric mode obtained at individual Strouhal numbers, as the available data does not allow to define a consistent scaling of the amplitude for the linear results. It can be concluded that the optimally forced linear wavepackets emit sound in a beam pattern that resembles the experimental 
measurements. However, the dominant beam angle in the linear model is larger by approximately $10^{\circ}$ than in the experiment, and a strong additional acoustic lobe around $90^{\circ}$ arises at $S t>0.8$, which is absent in the experimental data. Similar discrepancies were observed in the frequency response analysis of a DNS mean flow [10]. Notwithstanding, the overall agreement in the present case is clearly encouraging for further investigations into the linear modeling of jet dynamics and aeroacoustics. New experiments are under way that will facilitate a quantitative comparison of acoustic measurements with linear frequency response calculations.

Acknowledgements The authors are grateful to Dr. Guillaume Brès for making his LES database available. This work was supported by the Agence Nationale de la Recherche (ANR) under the "Cool Jazz" project, grant number ANR-12-BS09-0024. All instability calculations were performed using HPC resources of TGCC and CINES under the allocation 2015-2a6451 made by GENCI. The LES study was performed at Cascade Technologies, with support from the NAVAIR SBIR project, under the supervision of Dr. John T. Spyropoulos. The main LES calculations were carried out on DoD HPC systems in ERDC DSRC.

[1] E. Mollo-Christensen, Measurements of near field pressure of subsonic jets, Tech. rep., DTIC Document (1963).

[2] S. C. Crow, F. Champagne, Orderly structure in jet turbulence, Journal of Fluid Mechanics 48 (03) (1971) 547-591.

[3] J. B. Freund, Noise sources in a low-reynolds-number turbulent jet at mach 0.9, Journal of Fluid Mechanics 438 (2001) 277-305.

[4] D. Crighton, M. Gaster, Stability of slowly diverging jet flow, Journal of Fluid Mechanics 77 (02) (1976) 397-413.

[5] A. V. Cavalieri, D. Rodríguez, P. Jordan, T. Colonius, Y. Gervais, Wavepackets in the velocity field of turbulent jets, Journal of Fluid Mechanics 730 (2013) 559-592.

[6] P. Jordan, T. Colonius, Wave packets and turbulent jet noise, Annual Review of Fluid Mechanics 45 (2013) 173-195. 
[7] T. Suzuki, T. Colonius, Instability waves in a subsonic round jet detected using a near-field phased microphone array, Journal of Fluid Mechanics 565 (2006) 197-226.

[8] A. V. Cavalieri, P. Jordan, T. Colonius, Y. Gervais, Axisymmetric superdirectivity in subsonic jets, Journal of fluid Mechanics 704 (2012) 388-420.

[9] J. Jeun, J. W. Nichols, M. R. Jovanović, Input-output analysis of high-speed axisymmetric isothermal jet noise, Physics of Fluids (1994present) 28 (4) (2016) 047101.

[10] X. Garnaud, R. D. Sandberg, L. Lesshafft, Global response to forcing in a subsonic jet: instability wavepackets and acoustic radiation, AIAA paper 2013-4633.

[11] X. Garnaud, L. Lesshafft, P. Schmid, P. Huerre, The preferred mode of incompressible jets: linear frequency response analysis, Journal of Fluid Mechanics 716 (2013) 189-202.

[12] B. Wieneke, Stereo-piv using self-calibration on particle images, Experiments in Fluids 39 (2005) 267-280.

[13] F. Scarano, Iterative image deformation methods in piv, Meas. Sci. Tech. 13 (2002) R1-R19.

[14] J. Westerweel, F. Scarano, Universal outlier detection for piv data, Experiments in Fluids 39 (6) (2005) 1096-1100.

[15] K. Gudmundsson, T. Colonius, Instability wave models for the near-field fluctuations of turbulent jets, Journal of Fluid Mechanics 689 (2011) 97128.

[16] T. Troutt, D. McLaughlin, Experiments on the flow and acoustic properties of a moderate-reynolds-number supersonic jet, Journal of Fluid Mechanics 116 (1982) 123-156.

[17] G. A. Brès, J. Jaunet, M. Le Rallic, P. Jordan, T. Colonius, S. K. Lele, Large eddy simulation for jet noise: the importance of getting the boundary layer right, in: 21st AIAA/CEAS Aeroacoustics Conference, 2015, p. 2535. 
[18] P. Jordan, T. Colonius, G. Brès, M. Zhang, A. Towne, S. Lele, Modeling intermittent wavepackets and their radiated sound in a turbulent jet, in: Proceedings of the Summer Program, 2014, p. 241.

[19] J. Berland, C. Bogey, O. Marsden, C. Bailly, High-order, low dispersive and low dissipative explicit schemes for multiple-scale and boundary problems, Journal of Computational Physics 224 (2) (2007) 637-662.

[20] R. D. Sandberg, Governing equations for a new compressible navierstokes solver in general cylindrical coordinates, Monograph No. AFM07/07, School of Engineering Sciences, University of Southampton.

[21] W. Reynolds, A. Hussain, The mechanics of an organized wave in turbulent shear flow. part 3. theoretical models and comparisons with experiments, Journal of Fluid Mechanics 54 (02) (1972) 263-288.

[22] C. Mettot, D. Sipp, H. Bézard, Quasi-laminar stability and sensitivity analyses for turbulent flows: Prediction of low-frequency unsteadiness and passive control, Physics of Fluids (1994-present) 26 (4) (2014) 045112.

[23] P. Meliga, G. Pujals, E. Serre, Sensitivity of 2-d turbulent flow past a Dshaped cylinder using global stability, Physics of Fluids (1994-present) 24 (6) (2012) 061701.

[24] O. Tammisola, M. Juniper, Coherent structures in a swirl injector at $\mathrm{Re}=4800$ by nonlinear simulations and linear global modes, Journal of Fluid Mechanics 792 (2016) 620-657.

[25] S. Beneddine, D. Sipp, A. Arnault, J. Dandois, L. Lesshafft, Conditions for validity of mean flow stability analysis, Journal of Fluid Mechanics 798 (2016) 485-504.

[26] S. B. Pope, Turbulent flows, Cambridge University Press, 2000.

[27] A. Hanifi, P. J. Schmid, D. S. Henningson, Transient growth in compressible boundary layer flow, Physics of Fluids (1994-present) 8 (3) (1996) 826-837.

[28] O. Schmidt, A. Towne, T. Colonius, P. Jordan, V. Jaunet, A. V. Cavalieri, G. A. Brès, Super-and multi-directive acoustic radiation by linear 
global modes of a turbulent jet, in: 22nd AIAA/CEAS Aeroacoustics Conference, 2016, p. 2808.

[29] Y. B. Baqui, A. Agarwal, A. V. Cavalieri, S. Sinayoko, A coherencematched linear source mechanism for subsonic jet noise, Journal of Fluid Mechanics 776 (2015) 235-267.

[30] O. Semeraro, V. Jaunet, P. Jordan, A. V. Cavalieri, L. Lesshafft, Stochastic and harmonic optimal forcing in subsonic jets, in: 22nd AIAA/CEAS Aeroacoustics Conference, 2016, p. 2935. 